\subsection{2 \\ doi: 10.15789/2220-7619-2018-4-3.32 \\ EPIDEMIOLOGICAL ANALYSIS OF MEASLES \\ OUTBREAK IN GUINEA 2017-2018 \\ N'F. Magassouba \\ Gamal Abdel Nasser University of Conakry, Conacry, Republic of Guinea}

With the end of the largest ever known epidemic outbreak of Ebola in Guinea, since the beginning of 2016, comprehensive disease surveillance and response are under way. The five most common diseases, chosen according to their frequency in recent years, are monitored. These are viral hemorrhagic fevers, including Ebola and Yellow fever, cholera, meningitis, measles and poliomyelitis. Monitoring cases of measles shows that since the beginning of 2016 and despite the response organized in February 2016, confirmed cases are still being registered, and this occurs in several health districts.

The purpose of this paper was to describe the epidemiological profile of measles cases during the epidemic in 2016 and 2017. And also to study the results of the evaluation and the survey of immunization coverage in the framework of measles vaccination.

With the method of descriptive analysis, we developed a basic early warning system, a laboratory database and a national response plan

In 2016 (from 27 to 52 weeks of the year), 1304 suspected cases of measles were recorded, of which the blood was collected from 382 patients, which was $29.3 \%$. Of these, 379 $(99,2 \%)$ were admitted to the laboratory, among them 193 $(50.9 \%)$ were positive $(\operatorname{IgM}+=189$, indeterminate $=4)$.

In 2017 (from 1 to 9 weeks), 2.133 suspected cases of measles were recorded, blood was withdrawn from 549 patients $(25.73 \%)$. The laboratory received 443 samples (80.69) and $163(36.8 \%)$ were positive. Confirmed cases of measles continue to be recorded, despite the ongoing vaccination and campaign to fight the disease in the outbreaks. This epidemic affected 17 prefectures in the country from $38.73 \%$ of children is under the age of 5 . There is no difference between the sexes $(\mathrm{F}=50 \%, \mathrm{M}=50 \%)$. Coverage of vaccinations is 21 and $44 \%$, respectively, in cases of confirmation and without measles, with a total vaccination coverage of $35 \% .40 \%$ of cases of unconfirmed measles have $\operatorname{Ig} \mathrm{M}+$ to rubella. Mortality is low due to lack of information. Determining the cause of the persistence of measles will help to stop the epidemic.

\section{ASSESSMENT OF THE RISK OF MEASLES INFECTION IN HOSPITAL}

T. Mamaeva ${ }^{1}$, N. Zheleznova ${ }^{2}$, M. Naumova ${ }^{1}$

${ }^{1}$ Gabrichevsky Research Institute for Epidemiology and Microbiology, Moscow, Russia, ${ }^{2}$ St.Petersburg Pasteur Institute, St.Petersburg, Russia

There is no unambiguous assessment of the current epidemiological situation on measles. On the one hand, there is a decrease in morbidity, on the other - an increase in the number of seronegative women of childbearing age, thus affecting the measles incidence among the young children.

Assess the risk of measles infection in pregnant women, parturient women and newborns, as well as the need for serological examination of newborns aimed at the following anti-epidemic measures became possible with the introduction of infection in the hospital (maternity hospital). A total of 104 patients were examined in the laboratory. Blood serum was obtained from pregnant women (29), parturient women (46) and newborns (29) on the $2^{\text {nd }}$ day when the measles case in the obstetric ward was revealed.
The diagnosis of measles was confirmed by the ELISA (IgM) and PCR. The specific IgG antibodies were detected using the Anti-Measles Viruses ELISA (IgG) test system, Euroimmun (Germany).

In the study the seronegatives among the pregnant women consisted $31.0 \%$, and among the parturient women $34.8 \%$. In blood serum of the newborns IgG were detected in 21 patients $(72.4 \%)$. Taking into account that among the examined persons were 22 mother-child couples, it was possible to confirm the presence of maternal immunity. Thus, in the sera of 6 infants $\operatorname{IgG}$ antibodies were not detected and their mothers were also seronegative. At the same time in the sera of the other 16 infants from the seropositive mothers IgG antibodies were detected. The IgG titers varied from 0.2 to $3.0 \mathrm{IU} / \mathrm{ml}$ the mean value consisted $(0.70 \pm 0.45) \mathrm{IU} / \mathrm{mL}$ for infants and $(0.60 \pm 0.35) \mathrm{IU} / \mathrm{mL}$ for their mothers.

The data obtained show that in case of the risk of spreading the measles infection and deciding whether to perform a procedure for determining the specific IgG in the newborn's sera, it is more appropriate to carry out a serological examination of the parturient women due to the identity of the content of measles antibodies in the sera of infants and their mothers.

\subsection{4} doi: 10.15789/2220-7619-2018-4-3.34

\section{DEVELOPMENT OF PROTOTYPE OF UNIVERSAL INFLUENZA VACCINE BASED ON LIVE ATTENUATED INFLUENZA VACCINE VIRAL VECTOR}

D.A. Mezhenskaia ${ }^{1}$, T.S. Kotomina ${ }^{1}$, I.N. Isakova-Sivak ${ }^{1}$, V.A. Matyushenko ${ }^{1}$, A.S. Evsina ${ }^{1}$, M.C. Kim² ${ }^{2}$ S.M. Kang ${ }^{2}$, L.G. Rudenko ${ }^{1}$

${ }^{1}$ Institute of Experimental Medicine, St. Petersburg, Russia; ${ }^{2}$ Georgia State University, Atlanta, USA

Human influenza viruses are respiratory pathogens that cause annual epidemics and occasionally serious pandemic outbreaks. Seasonal influenza vaccination is the most effective way to control the spread of the disease; however it remains to be ineffective against pandemic influenza viruses due to the antigen mismatch. Therefore, the development of new universal vaccine with broad and durable effect is important issue for medical care. The extracellular domain of M2 protein (M2e) is highly conserved among all influenza A viruses and is widely used for generating broadly-reactive influenza vaccines. A new strategy of induction M2e-specific antibody is the expression of M2e tandem repeats in hemagglutinin (HA) molecule of live attenuated influenza vaccine (LAIV) used as viral vector. Recombinant LAIV viruses with chimeric HA proteins were generated by the means of reverse genetics. For that purpose BsmBI restriction site was inserted between signal peptide and HA1 subunit of HA genes of A/Switzerland/9715293/2013 (H3N2), A/Anhui/1/2013 (H7N9) or A/South Africa/3626/2013 (H1N1). Subsequently, four M2e tandem repeats were cloned into the inserted BsmBI cloning site. Recombinant LAIV viruses based on A/Leningrad/134/17/57 backbone were rescued by electroporation of Vero cells using Neon Transfection System (Invitrogen). All the LAIV-4M2e viruses actively replicated in eggs and preserved the temperature sensitive and cold-adapted phenotypes typical for LAIV viruses. Infectious virus titers were determined in eggs and MDCK cells incubated at different temperatures. Protective efficacy of new recombinant LAIVs against a panel of various influenza viruses was assessed in $\mathrm{BALB} / \mathrm{c}$ mouse model. In addition, the recombinant 